\title{
Instability of relativistic sheared jets and distinction between FRI and FRII sources
}

\author{
V. Urpin $\star$ \\ Departamento de Astronomia y Astrofizica, Universidad de Valencia, 46100 Burjassot, Spain \\ A.F. Ioffe Institute of Physics and Technology, 194021 St. Petersburg, Russia \\ Rceived 5 July 2001 / Accepted 18 December 2001

\begin{abstract}
We investigate the shear-driven instability of nonmagnetic relativistic jets with the bulk velocity, $V$, dependent on the cylindric radius, $r$. It is shown that instability can arise for any dependence of the velocity (or the Lorentz factor that is the same) on $r$. The shear-driven instability can effectively operate in the whole volume of a jet. The growth time can be shorter than that of the Kelvin-Helmholtz instability. The considered instability leads to a turbulization of jets and can account for a distiction between the jets in the FRI and FRII sources.
\end{abstract}

Key words. MHD - instabilities - ISM: jets and outflows - galaxies: jets

\section{Introduction}

The stability properties of jets has been a subject of analytical and numerical studies by many authors (see, for instance, Birkinshaw 1997 and references therein). These studies usually consider the jet as a gas beam with one bulk velocity and an interface made by a very narrow shear layer with the external medium. This model, however, can be too simplified because there exist some observational and theoretical evidence that the jet structure is more complex and different bulk velocities can be represented inside the jet (for more details see the discussion in Hanasz \& Sol 1996). For instance, Katz-Stone \& Rudnick (1994) have argued that jets in both FRI and FRII sources may have coaxial sheaths substantially wider than the inner jet itself. It is not immediately clear whether such sheaths are formed due to an interaction of the inner jet with the surrounding medium or have been ejected from the central engine. In fact, some models of jet formation predict the existence of a nonvanishing transverse gradient in the jet velocity (see, e.g., Melia \& Königl 1989; Königl \& Kartje 1994; Sol et al. 1989). Numerical simulations and analytical modelling of the propagation also indicate that the radial structure of jets can be more complex with a transition shear layer surrounding the core (Aloy 1999a, 1999b; Bicknell 1983; Kahn 1983). Obviously, the stability properties of such sheared jets may well be different from those of jets with the constant bulk velocity.

Large scale hydrodynamic instabilities can be the reason for the observed morphological complexity of jets, and this has motivated many analytical (see, e.g., Birkinshaw 1984, 1991; Hardee \& Norman 1988; Zhao et al. 1992;

\footnotetext{
* e-mail: urp@astro.ioffe.rssi.ru
}

Hanasz \& Sol 1996) and numerical (Hardee et al. 1992; Hardee et al. 1998; Bodo et al. 1998; Micono et al. 2000; Agudo et al. 2001) studies of the stability properties of jets. One possible mechanism of destabilization of astrophysical jets is often attributed to the well-known KelvinHelmholtz instability which in its classical formulation is the instability of a tangential discontinuity between two flows, generally of a different density (see, e.g., Landau \& Lifshitz 1978; Chandrasekhar 1981). Note that sometimes instability of a stratified fluid with continuously variable density and velocity is also called the KelvinHelmholtz instability (see, e.g., Chandrasekhar 1981). To distinguish between these cases we refer the "classical Kelvin-Helmholtz instability" as instability of the interface between two fluids. In real astrophysical jets the interface is likely smoothed, and its role is played by a transition layer which can be wider or narrower depending on the conditions. Obviously, the instability that can arise in such flows with a sheared transition layer is like the classical Kelvin-Helmholtz instability only if the tangential and normal lengthscales of perturbation exceed the characteristic thickness of the transition layer. In the opposite case, when the layer is very smooth and its thicknes is comparable to the jet radius, the instability has not very much in common with the classical Kelvin-Helmholtz instability. In the lack of stratification, the stability properties of such a jet with a smooth transition layer are probably closer to those of ordinary shear flows which are made turbulent due to shear stresses. This sort of instability is very common in hydrodynamics and leads to the turbulization of a flow at sufficiently large Reynolds numbers (see Landau \& Lifshitz 1978). We will call this instability shear-driven to distinguish it from the Kelvin-Helmholtz one. 
The classical Kelvin-Helmholtz instability is likely the dominating factor of destabilization in a simplified model of the jet with one bulk velocity and a narrow interface with the external medium. The analysis of such a model has been performed by many authors in either a linear regime (e.g., Blandford \& Pringle 1976; Ray 1981; Payne \& Cohn 1985; Zhao et al. 1992) or a nonlinear regime (Bodo et al. 1994; Koide et al. 1996; Nishikawa et al. 1997; Hardee et al. 1998). All these studies have shown that the Kelvin-Helmholtz instability can play an important role in the explanation of observed phenomena in astrophysical jets, such as jet disruption, limb-brightened features, surface filaments, etc. However, obviously the processes in such a simplified model cannot exhaust all the diversity of hydrodynamic phenomena in jets. For instance, a modification of the simplest model considered by Hanasz \& Sol (1996), who analysed a two-component jet model, shows very apparent differences with respect to the onecomponent model.

In the present paper we consider the stability properties of non-magnetized relativistic sheared jets. Note that the magnetic field likely plays a key role in the formation of astrophysical jets, and probably it can also influence the stability properties of sheared jets. The effect of the magnetic field is however beyond the scope of the present paper, and we are planning to address this problem elsewhere. The outline of this paper is as follows. In Sect. 2, we represent the basic equations governing the shear-driven instability in relativistic jets. In Sect. 3, we discuss the dispersion relation for short wavelength perturbations derived in a WKB-approximation. Instability of perturbations with long wavelengths is considered in Sect. 4. Finally, in Sect. 5, we discuss the possible role of the shear-driven instability as the mechanism of a distinction between jets in the FRI and FRII sources.

\section{Basic equations}

We model the jet by an infinitely long straight plasma cylinder of radius $R$. Plasma inside the jet has a velocity $\boldsymbol{V}=V(r) \boldsymbol{e}_{z}$ with respect to the ambient medium; $r, \varphi, z$ are the cylindrical coordinates with $\boldsymbol{e}_{r}, \boldsymbol{e}_{\varphi}, \boldsymbol{e}_{z}$ being the corresponding unit vectors. For the sake of simplicity, we assume that the temperature of plasma inside the jet is nonrelativistic. This assumption can likely be well justified at a sufficiently large distance from the central object where expansion and cooling process may cools down the jet plasma. Without loss of generality, we can consider the plasma density inside the jet, $\rho$, to be constant.

In relativistic hydrodynamics the continuity, momentum, and energy equations read (see, e.g., Weinberg 1972)

$\Gamma\left(\frac{\partial \rho}{\partial t}+\boldsymbol{v} \cdot \nabla \rho\right)+\left(\rho+\frac{p}{c^{2}}\right)\left[\frac{\partial \Gamma}{\partial t}+\nabla \cdot(\Gamma \boldsymbol{v})\right]=0$,

$\Gamma^{2}\left(\rho+\frac{p}{c^{2}}\right)\left[\frac{\partial \boldsymbol{v}}{\partial t}+(\boldsymbol{v} \cdot \nabla) \boldsymbol{v}\right]=-\nabla p-\frac{\boldsymbol{v}}{c^{2}} \frac{\partial p}{\partial t}$,

$\frac{\partial}{\partial t}\left(p n^{-\gamma}\right)+\boldsymbol{v} \cdot \nabla\left(p n^{-\gamma}\right)=0$ where $\Gamma=\left(1-v^{2} / c^{2}\right)^{-1 / 2}$ is the Lorentz factor, $p$ is the gas pressure and $n$ the number density in the fluid's rest frame; $\gamma$ is the adiabatic index. For plasma with a nonrelativistic temperature, we have $\rho \approx n m$ and $\rho \gg p / c^{2}$.

Our stability analysis is based on the linearized set of Eqs. (1)-(3). Small perturbations will be marked by the index 1; for unperturbed quantities subscripts will be omitted with the exception of vector components. The linearized continuity, momentum and energy equations are

$$
\begin{gathered}
\Gamma\left(\frac{\partial \rho_{1}}{\partial t}+\boldsymbol{v}_{1} \cdot \nabla \rho+\boldsymbol{V} \cdot \nabla \rho_{1}\right)+\rho\left[\nabla \cdot\left(\Gamma \boldsymbol{v}_{1}\right)\right. \\
\left.+\frac{\Gamma^{3}}{c^{2}} V\left(\dot{v}_{1 z}+V \frac{\partial v_{1 z}}{\partial z}\right)\right]=0 \\
\rho^{*}\left[\frac{\partial \mathbf{v}_{1}}{\partial t}+\left(\boldsymbol{v}_{1} \cdot \nabla\right) \boldsymbol{V}+(\boldsymbol{V} \cdot \nabla) \boldsymbol{v}_{1}\right]=-\nabla p_{1}-\frac{\boldsymbol{V}}{c^{2}} \dot{p}_{1}
\end{gathered}
$$

$$
\begin{array}{r}
\frac{\partial p_{1}}{\partial t}+\boldsymbol{v}_{1} \cdot \nabla p+\boldsymbol{V} \cdot \nabla p_{1}+\frac{\gamma p}{\Gamma}\left[\nabla \cdot\left(\Gamma \boldsymbol{v}_{1}\right)\right. \\
\left.+\frac{\Gamma^{3}}{c^{2}} V\left(\dot{v}_{1 z}+V \frac{\partial v_{1 z}}{\partial z}\right)\right]=0
\end{array}
$$

where $\rho^{*}=\Gamma^{2} \rho$. In Eq. (4) we took into account that $\Gamma_{1}=\Gamma^{3}\left(\boldsymbol{V} \cdot \boldsymbol{v}_{1}\right) / c^{2}$.

Since the unperturbed density, pressure and velocity do not depend on $t$ and the coordinates $\varphi$ and $z$, the dependence of all perturbations on these quantities can be taken in the form $\exp (i \omega t-i k z-i m \varphi)$. In the present paper, we consider axisymmetric perturbations with $m=0$. The dependence on $r$ has to be calculated from Eqs. (4)(6). Substituting this dependence into Eqs. (4) and (6), we can express the perturbation of pressure in terms of $\boldsymbol{v}_{1}$,

$$
p_{1}=\frac{i}{\sigma}\left\{\boldsymbol{v}_{1} \cdot \nabla p+\frac{\gamma p}{\Gamma}\left[\nabla \cdot\left(\Gamma \boldsymbol{v}_{1}\right)+i \sigma \frac{\Gamma^{3}}{c^{2}} V v_{1 z}\right]\right\}
$$

where $\sigma=\omega-k V(r)$. The quantity $k V$ is an advective frequency and has a kinematic origin. It always appears in a consideration of plane waves in a moving fluid if the wavevector has a component parallel to $\boldsymbol{V}$. The perturbations of a velocity can be expressed in terms of $p_{1}$ from the momentum Eq. (5) which for the considered perturbations has the form

$\rho \Gamma^{2}\left(i \sigma \boldsymbol{v}_{1}+v_{1 r} \frac{\mathrm{d} \boldsymbol{V}}{\mathrm{d} r}\right)=-i\left(\frac{\omega \boldsymbol{V}}{c^{2}}-i \nabla\right) p_{1}$.

Substituting expressions for $v_{1 r}$ and $v_{1 z}$ into Eq. (7) and taking into account that in the unperturbed state $\mathrm{d} p / \mathrm{d} r=$ 0 , we obtain the equation containing $p_{1}$ alone

$$
\begin{aligned}
p_{1}^{\prime \prime}+ & {\left[\frac{1}{r}+\frac{2 \Gamma^{2} V^{\prime}}{\sigma}\left(k-\frac{\omega V}{c^{2}}\right)\right] p_{1}^{\prime} } \\
& +\Gamma^{2}\left[\frac{\sigma^{2}}{c_{s}^{2}}-\left(k-\frac{\omega V}{c^{2}}\right)^{2}\right] p_{1}=0,
\end{aligned}
$$

where $c_{s}^{2}=\gamma p / \rho$ and $V^{\prime}=\mathrm{d} V / \mathrm{d} r$. Equation (9) represents the behaviour of small perturbations for any velocity profile, $V(r)$. It has been first derived by Birkinshaw (1984) 
in his stability analysis of two-component jets. If the jet is nonrelativistic and $V \ll c(\Gamma \approx 1)$, then the equation for $p_{1}$ simplifies very much

$p_{1}^{\prime \prime}+\left(\frac{1}{r}+\frac{2 V^{\prime} k}{\sigma}\right) p_{1}^{\prime}+\left(\frac{\sigma^{2}}{c_{s}^{2}}-k^{2}\right) p_{1}=0$.

This equation describes, for example, axisymmetric modes which can exist in supersonic nonrelativistic sheared jets.

For our purposes, it will be more convenient to use another form of the Eq. (9). Making a substitution

$p_{1}=\frac{1}{\sqrt{r}} \exp \left[-\int \frac{\Gamma^{2} V^{\prime}}{\sigma}\left(k-\frac{\omega V}{c^{2}}\right) \mathrm{d} r\right] f$,

Eq. (9) can be transformed into

$f^{\prime \prime}+q^{2}(r) f=0$,

where

$$
\begin{array}{r}
q^{2}=\frac{\Gamma^{2} \sigma^{2}}{c_{s}^{2}}-\frac{V^{\prime 2}}{\sigma^{2}}\left[2 k k_{1}\right. \\
\left.-\frac{\sigma^{2} \Gamma^{2}}{c^{2}}\left(1+\frac{\Gamma^{2} V^{2}}{c^{2}}\right)\right] \\
-\frac{k_{1}^{2}}{\Gamma^{2}}-\frac{\left(r V^{\prime}\right)^{\prime} k_{1}}{r \sigma}+\frac{1}{4 r^{2}},
\end{array}
$$

and $k_{1}=k-\sigma \Gamma^{2} V / c^{2}=\Gamma^{2}\left(k-\omega V / c^{2}\right)$. Note that $\Gamma\left(k-\omega V / c^{2}\right)$ is the $z$-component of a wavevector in the comoving frame.

Consider a very idealized model assuming that plasma moves with a relativistic velocity, $V(r) \approx c$, at $r \leq R$ and the Lorentz factor is large, $\Gamma(r) \gg 1$. The velocity is assumed to be varying slightly across the jet being close to $c$ at any $r \leq R$. On the contrary, the Lorentz factor, $\Gamma=\left(1-\bar{V}^{2} / c^{2}\right)^{-1 / 2}$, may vary within a very wide range. For the sake of simplicity, we assume that $\Gamma(r)$ varies monotonously and decreases with increasing $r$. For such a model, we have $\sigma \approx \omega-k c=$ const and $V^{\prime}=c^{2} \Gamma^{\prime} / V \Gamma^{3} \approx c \Gamma^{\prime} / \Gamma^{3}$. Substituting these expressions into Eq. (13), we obtain

$$
\begin{aligned}
q^{2}= & \frac{\Gamma^{2} \sigma^{2}}{c_{s}^{2}}\left[1-\left(\frac{k_{1} c_{s}}{\sigma \Gamma^{2}}\right)^{2}+\left(\frac{c_{s}}{2 r \sigma \Gamma}\right)^{2}\right]-\frac{2 k^{2} c^{2}}{\sigma^{2} \Gamma^{4}} \\
& \times\left\{\left(\frac{\Gamma^{\prime}}{\Gamma}\right)^{2} \frac{k_{1}}{k}+\left(\frac{\sigma \Gamma^{2}}{2 k c}\right) \frac{k_{1}}{k}\left(\frac{\Gamma^{\prime}}{r \Gamma}+\frac{\Gamma^{\prime \prime}}{\Gamma}-\frac{3 \Gamma^{\prime 2}}{\Gamma^{2}}\right.\right. \\
& \left.\left.-\frac{\Gamma^{\prime 2}}{\Gamma^{4}}\right)-2\left(\frac{\sigma \Gamma^{2}}{2 k c}\right)^{2}\left(\frac{\Gamma^{\prime}}{\Gamma}\right)^{2}\left(1+\frac{1}{\Gamma^{2}}\right)\right\}
\end{aligned}
$$

We are looking for a relatively fast instability with a growth rate greater than $c_{s} \max \left(k / \Gamma^{2}, 1 / 2 r \Gamma\right)$. In this case, the first term in the first paranthesis on the r.h.s. of Eq. (14) is larger than the second and third terms which can be neglected. The terms in the chain brackets of Eq. (14) are of a different order in the parameter $\sigma \Gamma^{2} / 2 c k$. This parameter can be small for real jet conditions (see below) because the growth time of hydrodynamic instability is usually much longer than the period of an electromagnetic wave. Therefore, we will neglect the terms of the first and second order in this parameter in
Eq. (14). Taking into account that $k_{1} \approx k$ at $|\sigma|<c k / \Gamma^{2}$, we can simplify the expression (14)

$q^{2} \approx \frac{\Gamma^{2} \sigma^{2}}{c_{s}^{2}}-\frac{2 c^{2} k^{2}}{\sigma^{2} \Gamma^{4}}\left(\frac{\Gamma^{\prime}}{\Gamma}\right)^{2}$.

If $\sigma^{2}$ is real and $\sigma^{2}>0$, the quantity $q^{2}$ has a positive maximum at the jet axis and decreases towards the boundary. For some values of $\sigma^{2}, q^{2}$ can even change the sign within the jet. On the contrary, if $\sigma^{2}<0$ (that corresponds to unstable modes), $q^{2}$ has a negative minimum at the axis and increases with an increase of $r$. Generally, in this case $q^{2}$ can change the sign as well.

Consider the solution of Eq. (12) in some particular cases.

\section{The instability in a WKB-approximation}

In this section, we consider the particular case when Eq. (12) can be solved by making use of a WKBapproximation which is well justified if $f$ has many knots in the radial direction (see, e.g., Landau \& Lifshitz 1981). Therefore, the condition of applicability of this approach is $|q| r>1$. Note, however, that a WKB-approximation yields qualitatively correct results even if the number of knots is not large, $n \sim 1$. In the region where $q^{2}>0$, the WKB-solution of Eq. (12) can be represented as

$f(r)=\frac{1}{\sqrt{q(r)}}\left[C_{1} \mathrm{e}^{i \int_{r_{0}}^{r} q \mathrm{~d} r^{\prime}}+C_{2} \mathrm{e}^{-i \int_{r_{0}}^{r} q \mathrm{~d} r^{\prime}}\right]$,

where $r_{0}$ is the so-called turning point at which $q^{2}=0$ and a WKB-approximation does not apply; $C_{1}$ and $C_{2}$ are constants that have to be choosen in such a way as to satisfy the boundary conditions. If $q^{2}>0$ everywhere within the jet then we can suppose $r_{0}=0$.

To obtain the eigenvalues and eigenfunctions of Eq. (12) one needs the boundary conditions for perturbations. The boundary condition at the axis of a jet is obvious: $f$ should be vanishing there. If $q^{2}$ has a turning point $r=r_{0}$ and $q^{2}<0$ at $0<r<r_{0}$, then this boundary condition implies that the solution has to go to 0 beyond the turning point, at $r<r_{0}$. It is known (see, e.g. Landau $\&$ Lifshitz 1981) that the solution (16) matches an exponentially decreasing solution beyond the turning point if $C_{1}=(C / 2) \exp (i \pi / 4)$ and $C_{2}=(C / 2) \exp (-i \pi / 4)$ where $C$ is constant. Hence, in this case, the solution (16) takes the form

$f(r)=\frac{C}{\sqrt{q(r)}} \cos \left(\int_{r_{0}}^{r} q\left(r^{\prime}\right) \mathrm{d} r^{\prime}+\frac{\pi}{4}\right)$.

The formulation of true boundary conditions at the outer boundary can face some problems but, fortunately, the WKB-approximation used in our treatment is well adopted for modes with a relatively small radial lengthscale. The properties of such modes are not sensitive to the particular choice of boundary conditions. Besides, the shear-driven instability is caused by the presence of an inhomogeneous velocity profile rather than the boundary 
condition and, due to this, is also not very sensitive to the latter. Therefore, the main conclusions of our analysis are qualitatively the same for any boundary conditions. To illustrate this point we can obtain and compare the WKB-solutions for different boundary conditions.

First, consider the case when the density in a surrounding medium is much larger than in the jet. In this case (see, e.g., Glatzel 1988; Wu \& Wang 1991), a much more dense surrounding medium plays the role of a wall and the perturbations of a radial displacement are vanishing at the outer boundary, $r=R$. As it follows from Eq. (8), the radial velocity vanishes if $\mathrm{d} p_{1} / \mathrm{d} r=0$ at $r=R$. Using the definition (11), we can rewrite this condition as

$\frac{\sigma \Gamma^{2} V}{k c^{2}}\left(f^{\prime}-\frac{f}{2 r}\right)-\frac{\Gamma^{\prime}}{\Gamma}\left(1-\frac{\sigma \Gamma^{2} V}{k c^{2}}\right) f=0$.

Taking into account that we consider the instability in the case $\sigma<k c / \Gamma^{2}$, the boundary condition ar $r=R$ can approximately be represented as

$f(R) \approx 0$.

With this boundary conditions, the dispersion equation reads

$\int_{r_{0}}^{R} q(r) \mathrm{d} r=\pi \alpha$,

where $\alpha=n+1 / 4, n$ is integer.

If the density of a jet is larger than that of a surrounding plasma, then perturbations of the pressure should be vanishing at the outer boundary, $p_{1}=0$ (Glatzel 1988; Wu \& Wang 1991), and we again obtain the condition (19). Therefore, with the accuracy in terms of the order of $\sigma \Gamma^{2} / c k$ the dispersion equation is the same for both considered limiting cases. Note that the main qualitative conclusions of our analysis will be unchanged even if we choose less realistic boundary conditions. For example, if we assume (without any physical motivation) that $\mathrm{d} f / \mathrm{d} r=0$ ar $r=R$ then instead of the dispersion Eq. (20) we obtain the same equation where $n+1 / 4$ is replaced by $n-1 / 4$. Since a WKB-approximation is valid if $n \gg 1$, this change will have a small impact on the result. This weak sensitivity to the boundary conditions reflects the fact that the shear-driven instability is caused mainly by shear stresses in the jet volume.

Equation (20) describes the eigenvalues of axisymmetric modes which can propagate in ultrarelativistic sheared jets. In the case $\sigma<c k / \Gamma^{2}$, the dispersion equation reads

$\int_{r_{0}}^{R}\left[\frac{\Gamma^{2}}{c_{s}^{2}} \sigma^{2}-\frac{2 c^{2} k^{2}}{\sigma^{2} \Gamma^{4}}\left(\frac{\Gamma^{\prime}}{\Gamma}\right)^{2}\right]^{1 / 2} \mathrm{~d} r=\pi \alpha$.

Using the mean value theorem, we can estimate the characteristic roots of Eq. (21) without specifying the velocity profile. We have

$\left[\frac{\Gamma_{*}^{2}}{c_{s}^{2}} \sigma^{2}-\frac{2 c^{2} k^{2}}{\sigma^{2} \Gamma_{*}^{4}}\left(\frac{\Gamma_{*}^{\prime}}{\Gamma_{*}}\right)^{2}\right]^{1 / 2}=\frac{\alpha \pi}{R_{1}}$, where $\Gamma_{*}$ is the value of the Lorentz factor at some point $r=r_{*}, R>r_{*}>r_{0}$, and $R_{1}=R-r_{0}$. Then, $\sigma^{2}$ is given by

$\sigma^{2}=\frac{c_{s}^{2}}{2 \Gamma_{*}^{2}}\left[\left(\frac{\pi \alpha}{R_{1}}\right)^{2} \pm \sqrt{\left(\frac{\pi \alpha}{R_{1}}\right)^{4}+\frac{8 c^{2} k^{2}}{c_{s}^{2} \Gamma_{*}^{2}}\left(\frac{\Gamma_{*}^{\prime}}{\Gamma_{*}}\right)^{2}}\right]$.

The first root (corresponding to the top sign) results only in a shift of the frequency of oscillations and is responsible for a drift of perturbations relatively to the mean flow. On the contrary, the second root (the bottom sign) leads to instability because $\sigma^{2}<0$ for this root. If $k \gg\left(\pi \alpha / 2 R_{1}\right)^{2}\left(c_{s} / c\right)\left(\Gamma / \Gamma^{\prime}\right)$, we have from Eq. (23)

$\sigma^{2} \approx \pm \frac{\sqrt{2} c_{s} c k}{\Gamma_{*}^{3}}\left(\frac{\Gamma_{*}^{\prime}}{\Gamma_{*}}\right)$,

and for the bottom sign we obtain

$\omega \approx k c \pm \frac{2^{1 / 4} i}{\Gamma_{*}^{3 / 2}} \sqrt{c_{s} c k\left|\Gamma_{*}^{\prime}\right| / \Gamma_{*}}$.

Our calculations apply if $\sigma$ satisfies the condition $|\sigma| \Gamma^{2} /$ $2 c k<1$. Substituning $\sigma$ from Eq. (24), we can rewrite this inequality as $c_{s} / c<2 \sqrt{2} k \Lambda / \Gamma_{*}$ where $\Lambda=\Gamma_{*} /\left|\Gamma_{*}^{\prime}\right|$. If the longitudinal wavelength of perturbations is comparable to the jet radius, $k \Lambda \sim 2 \pi$, we have $c_{s} / c<4 \sqrt{2} \pi / \Gamma_{*}$. This condition can well be fulfilled for many real relativistic jets.

The characteristic growth time of the unstable mode is

$\tau \approx \frac{\Gamma_{*}^{3 / 2}}{2^{1 / 4}} \sqrt{\frac{\Gamma_{*}}{c_{s} c k\left|\Gamma_{*}^{\prime}\right|}}$.

The growth time depends on the gradient of $\Gamma$ being shorter for a larger $\Gamma^{\prime}$. The instability arises faster for perturbations with a shorter axial wavelength but this dependence is relatively weak, $\tau \propto k^{-1 / 2}$. The growth time increses with an increase of the Lorentz factor therefore the instability can be suppressed in jets with very high $\Gamma$.

The characteristic growth length, $L=c \tau$, is given by

$L \approx 2^{-1 / 4} \Gamma_{*}^{3 / 2}\left(\frac{c}{c_{s}}\right)^{1 / 2}\left(\frac{\Gamma_{*}}{k\left|\Gamma_{*}^{\prime}\right|}\right)^{1 / 2}$.

For the axial wavelength comparable to the jet radius, $k \sim 1 / R$, and $\left|\Gamma_{*} / \Gamma_{*}^{\prime}\right| \sim R$, the instability can manifest itself at the distance

$L \sim R \Gamma_{*}^{3 / 2} \sqrt{c / c_{s}}$

from the central object. Obviously, the growth length becomes longer and longer with an increase of the Lorentz factor, and in highly relativistic jets the instability developes only in the most removed regions or does not appear at all if the length of a jet is smaller than $L$.

If $n$ is very large and $k \ll\left(\pi \alpha / 2 R_{1}\right)^{2}\left(c_{s} / c\right)\left(\Gamma / \Gamma^{\prime}\right)$ then, from Eq. (23), we obtain the following expression for $\sigma^{2}$ corresponding to unstable modes

$\sigma^{2} \approx-\frac{2 c^{2} k^{2} R_{1}^{2}}{\pi^{2} \Gamma_{*}^{4} n^{2}}\left(\frac{\Gamma_{*}^{\prime}}{\Gamma_{*}}\right)^{2}$,

and the growth rate decreases with increasing $n$. 


\section{The instability of long wavelength perturbations}

The instability can arise not only in small radial scales, as required by a WKB-approximation, but also in scales comparable to the jet radius. Consider the case of a relatively smoothly varying $\Gamma$-profile when we can obtain the analytical solution of Eq. (12),

$\Gamma(r)=\Gamma_{0}\left(\frac{b}{b+r}\right)^{1 / 3}$,

where $\Gamma_{0}$ is the value of $\Gamma$ at the jet axis, and $b$ characterises the width of a distribution of $\Gamma$. At the jet surface, we have $\Gamma(R)=\Gamma_{0}[b /(b+R)]^{1 / 3}$.

For such $\Gamma(r)$, Eq. (12) with $q^{2}$ given by Eq. (15) transforms into

$f^{\prime \prime}+\left(\frac{b}{b+r}\right)^{2 / 3}\left(\frac{\sigma^{2} \Gamma_{0}^{2}}{c_{s}^{2}}-\frac{2 c^{2} k^{2}}{9 \sigma^{2} \Gamma_{0}^{4} b^{2}}\right) f=0$.

Introducing the new variable, $x=r+b$, and denoting

$\alpha=b^{2 / 3}\left(\frac{\sigma^{2} \Gamma_{0}^{2}}{c_{s}^{2}}-\frac{2 c^{2} k^{2}}{9 \sigma^{2} \Gamma_{0}^{4} b^{2}}\right)$,

we can rewrite Eq. (31) as

$\frac{\mathrm{d}^{2} f}{\mathrm{~d} x^{2}}+\frac{\alpha}{x^{2 / 3}} f=0$.

The solution of this equation can be expressed in terms of the Bessel functions,

$f=\sqrt{x}\left[A_{1} J_{3 / 4}\left(3 \sqrt{\alpha} x^{2 / 3} / 2\right)+A_{2} Y_{3 / 4}\left(3 \sqrt{\alpha} x^{2 / 3} / 2\right)\right]$,

where $A_{1}$ and $A_{2}$ are constant. The dispersion equation can easily be obtained from the boundary onditions. We have

$Y_{3 / 4}(\xi) J_{3 / 4}(\lambda \xi)=J_{3 / 4}(\xi) Y_{3 / 4}(\lambda \xi)$,

where $\xi=3 \sqrt{\alpha} b^{2 / 3} / 2$ and $\lambda=(1+R / b)^{2 / 3}>1$. All the roots of this equation are real and simple (see, e.g., Abramowitz \& Stegun 1970). The asymptotic expansion of the $s$ th zero is

$$
\begin{aligned}
\xi_{s}= & \frac{s \pi}{\lambda-1}+\frac{5(\lambda-1)}{32 \lambda \pi s}-\frac{25(\lambda-1)^{3}}{6144 \pi^{3} \lambda^{3} s^{3}} \\
& \times\left(19 \lambda^{2}+25 \lambda+19\right)+\ldots
\end{aligned}
$$

The frequency, $\sigma$, corresponding to the $s$ th zero, satisfies the equation

$$
\frac{\sigma^{2} b^{2} \Gamma_{0}^{2}}{c_{s}^{2}}-\frac{2 c^{2} k^{2}}{9 \sigma^{2} \Gamma_{0}^{4}}=\frac{4}{9} \xi_{s}^{2}
$$

Solving for $\sigma^{2}$, we obtain the expression

$\sigma^{2}=\frac{2 c_{s}^{2} \xi_{s}^{2}}{9 b^{2} \Gamma_{0}^{2}}\left[1 \pm \sqrt{1+\frac{9}{2 \xi_{s}^{2} \Gamma_{0}^{2}}\left(\frac{c}{c_{s}}\right)^{2} b^{2} k^{2}}\right]$

which is the analogy of Eq. (23). One of the roots of this equation represents an unstable mode with $\sigma^{2}<0$. If $\left(3 / \sqrt{2} \xi_{s}^{2} \Gamma_{0}\right)\left(c / c_{s}\right) b k>1$, the expression for $\sigma^{2}$ simplifies, and we have

$\sigma^{2} \approx \pm \frac{\sqrt{2} c_{s} c k}{3 \Gamma_{0}^{3} b}$

Then,

$\omega \approx k c \pm \frac{2^{1 / 4} i}{3^{1 / 2} \Gamma_{0}^{3 / 2}} \sqrt{c_{s} c k / b}$,

and the characteristic growth time of the unstable mode is given by

$\tau \approx 1.45 \Gamma_{0}^{3 / 2} \sqrt{b / c_{s} c k}$

The corresponding growth length is

$L \approx 1.45 \Gamma_{0}^{3 / 2} \sqrt{\frac{c b}{c_{s} k}} \approx 0.6 \Gamma_{0}^{3 / 2} R\left(\frac{b \lambda}{R^{2}}\right)^{1 / 2}\left(\frac{c}{c_{s}}\right)^{1 / 2}$,

where $\lambda=2 \pi / k$ is the axial wavelength of perturbations. The dependence of $\tau$ and $L$ on the parameters is qualitaively the same as for short wavelength modes discussed in the previous section.

\section{Discussion}

We considered the instability that can arise in relativistic sheared jets. In our simplified model, the particular mechanism of the formation of a shear layer is unimportant. The instability considered is probably only the limiting case of various shear-driven instabilities that can exist in supersonic jets. This sort of instability has already been considered by a number of authors for nonrelativistic flows and for some particular velocity profiles. Blumen et al. (1975) and Drazin \& Davey (1977) examined the hyperbolic tangent profile in the case of compressible fluid and showed that this profile leads to instability. A linear supersonic shear layer has been considered in detail by Glatzel (1988) who obtained that both Kelvin-Helmholtz modes and sonic modes are responsible for instability. This allows us to speculate that shear-driven instabilities probably play an important role in all supersonic jets. In our model, the instability is caused by the radial dependence of the Lorenz factor that is a particular case of shear.

The physical reasons causing the shear-driven instability in a compressible flow are rather simple. Let us assume that at some instant of time a small perturbation of the radial velocity has been created, $v_{1 r}$. Due to the presence of shear, the inertial force can generate from $v_{1 r}$ some motion in the $z$-direction. Since the mean flow is supersonic but the gas temperature and pressure are assumed to be nonrelativistic, the pressure plays practically no role in the momentum transfer in the $z$-direction. Therefore, the longitudinal acceleration is almost entirely determined by the inertial force,

$\frac{\mathrm{d} v_{1 z}}{\mathrm{~d} t} \approx-v_{1 r} \frac{\mathrm{d} V}{\mathrm{~d} r}$ 
(see the $z$-component of the momentum Eq. (8)), d/d $t=$ $\partial / \partial t+V \partial / \partial z$. If we denote the characteristic local time of the process as $\tau_{0}$ then we can estimate $v_{1 z}$ as $\sim V^{\prime} \tau_{0} v_{1 r}$. In a compressible gas both the radial and longitudinal motions lead generally to nonuniformities of the density, $\rho_{1}$. For the conditions under consideration the effect of $v_{1 z}$ is typically more pronounced and longitudinal motions form density nonuniformities in the $z$-direction. Therefore, we have from the continuity condition

$\rho_{1} \sim \rho k \tau_{0} v_{1 z} \sim \rho k \tau_{0}^{2} V^{\prime} v_{1 r}$

where $k$ is again the longitudinal wave vector. Perturbations of the density are accompanied by those of the pressure. In the adiabatic case, these perturbations are related by $p_{1}=c_{s}^{2} \rho_{1}$. Then,

$p_{1} \sim \rho k c_{s}^{2} \tau_{0}^{2} V^{\prime} v_{1 r}$.

In its turn, perturbations of the pressure depend on $r$ and can change the radial velocity due to a pressure force. The change of a radial velocity after a short time interval, $\Delta t$, is given by

$\Delta v_{1 r} \sim \frac{1}{\rho \Gamma^{2}} \frac{\partial p_{1}}{\partial r} \Delta t$

where the coefficient before $\Delta t$ is the acceleration caused by a pressure force. The radial pressure gradient is mainly determined by a strong dependence of $\tau_{0}$ on $r$ since we have $\partial\left(1 / \tau_{0}\right) / \partial r \sim k V^{\prime}$. Substituting this expression into Eq. (46), we obtain that the change of radial velocity is

$\Delta v_{1 r} \sim \frac{k^{2} c_{s}^{2} V^{\prime 2}}{\Gamma^{2}} \tau_{0}^{3} v_{1 r} \Delta t$

Again, estimating $\Delta v_{1 r} / \Delta t$ as $v_{1 r} / \tau_{0}$, we obtain the following estimate for the timescale of instability

$\frac{1}{\tau_{0}} \sim \sqrt{\frac{k c_{s} V^{\prime}}{\Gamma}}$

in a good agreement with Eqs. (26) and (41). As it is seen from this explanation shear stresses and compressibility are the most important underlying physical effects resulting in the instability. Note that compressibility can be crucial also for some other instability mechanisms proposed for supersonic jets. For instance, Payne \& Cohn (1985) considered the instability caused by reflection of sound waves from the jet boundary. After the sound waves travelling towards the jet have been reflected off the jet axis, they propagate outward to the boundary, striking it where the expansion is maximum. Not all the waves striking the boundary escape the jet; a significant fraction is reflected back into the jet once again. Since perturbations are periodic, the ingoing waves beginning their second cycle are aided by the perturbation of the jet cross-section, one wavelength downstream of the first. The resonant nature of this instability arises bacause a given perturbation is reinforced by the perturbation upstream of it. Clearly, that the mechanism considered in our paper is qualitatively different from that proposed by Payne \& Cohn (1985).
The fact of instability itself does not depend very much on the particular shape of a velocity profile. As it follows from Eq. (25), instability can arise for any profile. The characterisic growth time and growth length depend, however, on shear being shorter for a sharper dependence of $V$ on $r$. Note that only perturbations with a nonvanishing axial wavevector, $k \neq 0$, can be unstable, and the growth rate is larger for a shorter wavelength.

The principle difference between the considered instability and the well-studied Kelvin-Helmholtz instability is that the latter develops in the boundary layer whereas the shear-driven instability arises in the volume of a jet. It is also important to emphasise that the growth rate of the relativistic shear-driven instability is approximately by a factor $\sqrt{c / c_{s}}$ larger than that of the Kelvin-Helmholtz instability and, correspondingly, the growth length is by the same factor shorter (compare, for instance, to Hardee et al. 1998). Due to this, the considered instability can be an efficient mechanism for the generation of turbulent motions inside the jet. In its turn, turbulence in highly supersonic flows can be responsible for various physical processes such as generation of the magnetic field and synchrotron emission, formation of shocks etc.

As follows from Eqs. (27) and (41), an increase of $\Gamma$ makes the growth time longer, and the instablity has to be less efficient in highly relativistic jets. Under certain conditions, it can be possible that the growth length of instability, $L$, is longer than the length of a jet. In this case, the jet can remain more or less laminar up to the head region. Note also that the growth length depends on the temperature of plasma inside the jet being longer for cooler jets.

The dependence of the growth rate on the Lorentz factor and the temperature can explain the difference in properties of jets in extragalactic radio sources. Observations suggest that the flow speed in these jets is relativistic and more or less laminar on parsec scales in both the powerful FRII sources and the weaker FRI sources (Kellerman \& Pauliny-Toth 1981; Ghisellini et al. 1993). In contrast, on the kiloparsec scale, jets in FRI sources are of a lower power and more turbulent compared to jets in FRIIs which are more stable and laminar (see, e.g., Kaiser \& Alexander 1997). A possible explanation suggested by a number of authors (see Bridle 1984; Sol et al. 1989) is the physical distinction of flows on the large and small scales. It seems the hypothesis that jets in FRI sources decelerate from relativistic speeds on kiloparsec scales is consistent with many of their observed properties (Laing 1994). The physical mechanisms behind this deceleration are not immediately clear, but it has been emphasised by Laing (1994) that velocity gradients across and along relativistic jets may radically modify their appearence. The instability considered in the present paper can be one of the driving forces caused a deceleration (and even destruction) of jets.

The scenario can be the following. All jets in extragalactic radio sources start out basically with presumably relativistic but slightly different bulk velocities. After that, 
turbulence rapidly growing due to the shear-driven instability in weaker and hotter jets destroys the laminar structure and slows down jets. Such sources should have an appearences of the FRI type. The flow in stronger and cooler jets stays mostly laminar because the instability arises on a longer time scale. At intermediate distances from the central engine, before the weaker jets have become entirely turbulent, the appearences of both weak and strong jets should be similar which is confirmed by observations (Laing 1993). Note that in our model not only the Lorentz factor determines the growth rate of instability but the temperature as well. In fact, a hotter jet with a larger Lorentz factor can be less stable than a cooler jet with a smaller $\Gamma$. Qualitatively the same dependence on the temperature can be obtained if the jet slows down by accumulating gas from the surroundings (Bowman et al. 1996). All else being equal, initially hotter jets are shown by these authors to have lower energy fluxes than cooler flows, indicating that the former are more closely related to FRI sources and the latter to FRIIs.

It is not clear from observations, however, at which scale jets become turbulent and decelerate. As it was mentioned, most likely, these scales lie between the pc and kpc scales. We give an estimate of the growth length for the jet model with the radius $\sim 50 \mathrm{ps}$ and the Lorentz factor $\Gamma_{0}=5$. We assume that $c_{s} \sim 0.1 c$, that corresponds to the temperature $\sim 10^{11} \mathrm{~K}$. For the chosen values of parameters, the quantity $\sigma \Gamma^{2} / 2 c k$ is approximately equal to $0.08 \sqrt{\lambda / b}$ if we use the expression (39) for $\sigma$. Obviously, for $\lambda \leq b$ this quantity is actually small and the expression (15) for $q^{2}$ is well justified. Therefore, we can use the estimate (42) for the growth length. For perturbations with $\lambda=R$ we obtan $L \approx 1.2 \sqrt{b / R}$ kpc. This estimate gives a reasonable value for the growth length of instability in FRI jets. Note that, according to our model, perturbations with a shorter wavelength should grow faster, therefore small scale turbulent structures can be observable even at intermediate distances.

Acknowledgements. The author is grateful to Professors J. M. Ibañez and J. M. Marti for helpful discussions and stimulating interest in this work. This research was supported in part by the Russian Foundation of Basic Research and Deutsche Forschungsgemeinschaft (grant 00-02-04011). The author also thanks Ministerio de Educacion, Culture y Deporte of Spain for the financial support under the grant SAB1999-0222.

\section{References}

Abramowitz, M., \& Stegun, I. 1970, Handbook of Mathematical Functions (New York: Dover Publications, Inc.)

Agudo, I., Gomez, J. L., Marti, J. M., et al. 2001, ApJ, 549, L183

Aloy, M. A., Ibáñez, J. M., Marti, J. M., \& Müller, E. 1999a, ApJS, 122, 151
Alloy, M. A., Ibáñez, J. M., Marti, J. M., \& Müller, E. 1999b, ApJ, 523, L125

Bicknell, G. V. 1983, PASAu, 5, 130

Birkinshaw, M. 1984, MNRAS, 208, 887

Birkinshaw, M. 1991, MNRAS, 252, 505

Birkinshaw, M. 1997, In Advanved Topics on Astrophysical and Space Plasmas, ed. E. Gouveia Dal Pino, A. Peratt, G. Megina Tancro, \& A. Chian (Dordrecht: Kluwer)

Blandford, R., \& Pringle, J. E. 1976, MNRAS, 176, 443

Blumen, W., Drazin, P., \& Billings, D. 1975, J. Fluid Mech., 71, 305

Bodo, G., Massaglia, S., Ferrari, A., \& Trussoni, E. 1994, A\&A, 283, 655

Bodo, G., Rossi, P., \& Massaglia, S. 1998, A\&A, 333, 1117

Bowman, M., Leahy, J., \& Komissarov, S. 1996, MNRAS, 279, 899

Bridle, A. 1984, In Physics of Energy Transport in Extragalactic Radio Sources, ed. A. Bridle, \& J. Eilek (Green Bank: NRAO)

Chandrasekhar, S. 1981, Hydrodynamic and Hydromagnetic Stability (New York: Dover Publication, Inc.)

Drazin, P., \& Davey, A. 1977, J. Fluid Mech., 82, 355

Hanasz, M., \& Sol, H. 1996, A\&A, 315, 355

Hardee, P., \& Norman, M. 1988, ApJ, 334, 70

Hardee, P., Cooper, M., Norman, M., \& Stone, J. 1992, ApJ, 399, 478

Hardee, P., Rosen, A., Hughes, P., \& Comer Duncan, G. 1998, ApJ, 500, 599

Ghisellini, G., Padovani, P., Celotti, A., \& Maraschi, L. 1993, ApJ, 407, 65

Glatzel, W. 1988, MNRAS, 231, 795

Kahn, F. D. 1983, MNRAS, 202, 553

Kaiser, C., \& Alexander, P. 1997, MNRAS, 286, 215

Katz-Stone, D., \& Rudnick, L. 1994, ApJ, 426, 116

Kellermann, K., \& Pauliny-Toth, I. 1981, ARA\&A, 19, 373

Koide, S., Nishikawa, K.-I., \& Mutel, R. I. 1996, ApJ, 463, L71

Königl, A., \& Kartje, J. 1994, ApJ, 434, 446

Laing, R. A. 1993, in Astrophysical Jets, ed. D. Burgarella, M. Livio, \& C. O'Dea (Cambridge: Cambridge University Press)

Laing, R. a. 1994, in The First Stromlo Symposium: The Physics of Active Galaxies, ed. G. V. Bicknell, M. Dopita, \& P. Quinn (ASP Conf. Ser.), 54

Landau, L., \& Lifshitz, E. 1978, Fluid Mechanics (Moscow: Nauka)

Landau, L., \& Lifshitz, E. 1981, Quantum Mechanics (Moscow: Nauka)

Melia, F., \& Königl, A. 1989, ApJ, 340, 162

Micono, M., Bodo, G., Massaglia, S., et al. 2000, A\&A, 360, 795

Nishikawa, K.-I., Koide, S., Sakai, J.-L., et al. 1997, ApJ, 483, L45

Payne, D., \& Cohn, H. 1985, ApJ, 291, 635

Ray, T. 1981, MNRAS, 196, 195

Sol, H., Pelletier, G., \& Asseo, E. 1989, MNRAS, 237, 411

Weinberg, S. 1972, Gravitation and Cosmology (New York: Wiley)

Wu, D., \& Wang, D. 1991, MNRAS, 250, 760

Zhao, J.-H., Burns, J., Hardee, P., \& Norman, M. 1992, ApJ, 387,69 